\title{
Analyse didactique des praxéologies de modélisation mathématique à l'école : une étude de
}

\section{cas}

Didactic analysis of mathematical modelling practices in primary school: a study case

\section{Floriane Wozniak}

\section{OpenEdition \\ Journals}

Édition électronique

URL : http://journals.openedition.org/educationdidactique/1471

DOI : 10.4000/educationdidactique.1471

ISSN : 2111-4838

Éditeur

Presses universitaires de Rennes

\section{Édition imprimée}

Date de publication : 30 octobre 2012

Pagination : $65-88$

ISBN : $978-2-7535-1984-8$

ISSN : 1956-3485

\section{Référence électronique}

Floriane Wozniak, « Analyse didactique des praxéologies de modélisation mathématique à l'école : une étude de cas », Éducation et didactique [En ligne], 6-2 | octobre 2012, mis en ligne le 30 octobre 2014 consulté le 08 décembre 2020. URL : http://journals.openedition.org/educationdidactique/1471 ; DOI : https://doi.org/10.4000/educationdidactique.1471 


\title{
ANALYSE DIDACTIQUE DES PRAXÉOLOGIES DE MODÉLISATION MATHÉMATIQUE À L'ÉCOLE : \\ UNE ÉTUDE DE CAS
}

Floriane Wozniak (IUFM d'Alsace - Université de Strasbourg, IRIST EA 3424)

\begin{abstract}
Résumé : Considérant l'évolution du curriculum mathématique vers un enseignement des pratiques de modélisation et de la démarche d'investigation, nous nous intéressons ici aux conditions et contraintes de leur diffusion à l'école primaire. Cette question est abordée à travers l'observation des praxéologies spontanées de modélisation d'un professeur dans une classe ordinaire de CM2 (enfants de dix ou onze ans). L'analyse est conduite à partir de la Théorie Anthropologique du Didactique et reprend le modèle du processus de modélisation de Chevallard (1989) interrogé par la notion de problématisation de Orange (2005). Au-delà des besoins de savoirs relatifs à la modélisation mathématique, l'étude de cas permet d'illustrer la nécessité de mettre en œuvre des praxéologies didactiques spécifiques de la démarche d'investigation. Pratiques qui révèlent la généricité des savoirs sous la contingence des techniques utilisées pour résoudre un problème singulier posé par le réel.
\end{abstract}

Mots clés : théorie anthropologique du didactique, problématisation, mathématiques, école primaire, modélisation.

Floriane Wozniak

\section{Introduction}

En 2007 parait le rapport d'un groupe d'experts commandité par les commissaires européens responsables de la recherche, de l'éducation et de la culture. Prenant acte que « de nombreuses études ont mis en évidence une alarmante perte d'intérêt des jeunes pour les études scientifiques et mathématiques " (Rocard, Csermely, Jorde, Lenzen, Walberf-Henrikson, Hemmo, 2007, p. 5), ils souhaitent que des propositions soient faites à la Commission Européenne sur l'enseignement scientifique. Définissant la science en page 5 comme « tout système de connaissances qui tente de modéliser la réalité objective », les rapporteurs affirment que:

« Renverser la pédagogie utilisée pour enseigner les sciences à l'école, en la faisant passer de méthodes essentiellement déductives à des méthodes basées sur l'investigation permet d'augmenter l'intérêt des jeunes pour les sciences. » (Ibid., 2007, p. 2).

Cette proposition de changement de paradigme scolaire est explicitée en ces termes:

« L'enseignement basé sur les problèmes désigne un environnement d'apprentissage dans lequel les problèmes guident l'apprentissage. Autrement dit, l'apprentissage commence par un problème à résoudre et le dit problème est posé de façon à obliger les enfants à acquérir de nouvelles connaissances avant même l'étape de résolution proprement dite. Plutôt que de rechercher une réponse correcte unique, les enfants interprètent le problème, recueillent les informations nécessaires, identifient les solutions possibles, évaluent les différentes options disponibles et formulent des conclusions. L'enseignement des sciences basé sur l'investigation constitue une approche basée sur les problèmes, mais avec une dimension supplémentaire étant donné l'importance accordée à l'approche expérimentale. » (Ibid, p. 10).

La reconnaissance du rôle et de la place de la résolution de problèmes à l'école primaire n'est cependant pas une question nouvelle comme en attestent deux des neufs documents d'accompagnement ou d'application des programmes instaurés en 2002 en France: «Les problèmes pour chercher » et « Résolution de problèmes et apprentissage ». Les auteurs y attribuaient quatre fonctions aux problèmes: la construction d'une nouvelle connaissance; le réinvestissement de connaissances déjà travaillées; la mobilisation de plusieurs catégories de connaissances; le développement des capacités à chercher. Avec le renouvellement des programmes en 2007 puis 2008, ces documents sont devenus institutionnellement obsolètes. Néanmoins au cycle 3 de l'école élémentaire - enfants de huit à onze ans - le préambule de présentation du programme de mathématiques actuellement en vigueur réaffirme que « la pratique des mathématiques développe le goût de la recherche et du raisonnement, l'imagination et les 
capacités d'abstraction, la rigueur et la précision » (Ministère de l'Éducation Nationale, 2008, p. 22). Avant de conclure que « La maîtrise des principaux éléments mathématiques aide à agir dans la vie quotidienne et prépare la poursuite d'études au collège. » (Ibid, p. 22).

Ce qui semble nouveau, c'est l'insistance à aborder un certain type de problèmes, comme l'illustrent les trois citations de la page suivante du même document:

« La résolution de problèmes liés à la vie courante permet d'approfondir la connaissance des nombres étudiés, de renforcer la maîtrise du sens et de la pratique des opérations, de développer la rigueur et le goût du raisonnement $»[\ldots]$

« La résolution de problèmes concrets contribue à consolider les connaissances et capacités relatives aux grandeurs et à leur mesure, et à leur donner sens. À cette occasion des estimations de mesure peuvent être fournies puis validées. » [...]

"Les capacités d'organisation et de gestion des données se développent par la résolution de problèmes de la vie courante ou tirés d'autres enseignements. » (Ibid, p. 23).

Nous interprétons une telle insistance à évoquer cette catégorie de problèmes « concrets » et « liés à la vie courante » comme la réponse de l'institution scolaire aux questions soulevées par les résultats des élèves français de quinze ans aux évaluations du Programme for International Student Assessment (PISA) organisées par l'Organisation de Coopération et de Développement Economiques (OCDE). Dans ces évaluations où les problèmes sont essentiellement issus de la vie quotidienne « les résultats français sont supérieurs à la moyenne de l'OCDE lorsqu'il s'agit d'exercices purement scolaires, mais cela n'est pas le cas lorsque la situation nécessite une prise d'initiative » (Bourny, Dupé, Robin, \& Rocher, 2001, p. 4).

Il semble donc qu'un nouveau rapport institutionnel aux objets de savoirs mathématiques s'inscrive dans un rapport au monde qui sorte les mathématiques de l'autarcie dans laquelle elles s'étaient installées pour s'ouvrir à des organisations mathématiques mixtes, mathématiques citoyennes parce qu'utiles pour penser sa place et agir dans la cité. Ce mouvement curriculaire conduit ainsi à l'intégration dans l'enseignement de la modélisation mathématique. Mais si l'institution scolaire souhaite instaurer un nouveau paradigme fondé sur le questionnement du monde, il peut être fécond d'établir les conditions qui faciliteront un tel changement et corrélativement de déceler les contraintes qui pèsent voire empêchent un tel changement. Ce type de questionnement s'inscrit dans ce que Chevallard (2011) appelle la problématique primordiale:

«Étant donné un projet d'activité dans lequel telle institution ou telle personne envisage de s'engager, quel est, pour cette institution ou cette personne, l'équipement praxéologique qui peut être jugé indispensable ou simplement utile dans la conception et l'accomplissement de ce projet? » (Chevallard, 2011, p. 98).

Or aborder la question du changement de rapport institutionnel au savoir selon une telle problématique conduit à dresser un état des lieux des praxéologies existantes. C'est ce que nous nous proposons de faire dans ce texte qui présente l'analyse des pratiques d'enseignement d'un professeur dans une « classe ordinaire » du point de vue des savoirs mathématiques mis en jeu. Si une seule étude de cas ne permet pas de réaliser un état des lieux, elle permet d'en poser les prémisses par les questions qu'elle pose. Pour réaliser cette étude qualitative nous utilisons la Théorie Anthropologique du Didactique, en cohérence avec la définition qu'elle donne de la didactique: « science des conditions et des contraintes de la diffusion (et de la non-diffusion) des praxéologies au sein des institutions de la société »(Chevallard, 2010, p. 140).

Dans un premier temps nous expliciterons ce que nous appelons des praxéologies de modélisation. Notre objet d'étude portant sur les praxéologies, nous en présenterons une typologie en fonction du rôle et de la place du discours technologique à partir des travaux de Assude, Mercier, et Sensevy (2007). L'analyse a priori des praxéologies mathématiques permettant la résolution du problème que des élèves de CM2 auront à étudier nous conduira à interroger notre cadre théorique avec la notion de problématisation de Orange (2005). Après une présentation des conditions de recueil de notre observation, nous présenterons alors une analyse didactique détaillée de 
la séance observée du point de vue des savoirs mathématiques. Il ne s'agira pas de mesurer la cohérence entre la préparation et la conduite de classe du professeur mais de dégager au-delà de ce qui est fait, ce qui pourrait être, voire ce qui devrait être, pour que vivent des praxéologies de modélisation telles que nous les définissons dans ce texte. Ceci nous permettra, en conclusion, de revenir sur les besoins praxéologiques des professeurs pour que vivent dans les classes de mathématiques les pratiques de modélisation.

\section{La Théorie Anthropologique du Didactique comme cadre théorique}

La Théorie Anthropologique du Didactique (TAD) considère que les mathématiques, comme n'importe quelle activité humaine, se produisent, se diffusent, se pratiquent, s'enseignent ou s'apprennent par des personnes au sein d'institutions sociales dont elles sont les sujets. Ainsi, par exemple, professeur et élèves "font " des mathématiques dans l'institution que forme une classe de l'école primaire aujourd'hui en France. Mais s'ils sont sujets d'une même institution, ils n'y occupent pas la même position, notamment par rapport aux savoirs scolaires. C'est que toute institution définit un rapport institutionnel aux objets, fait de la manière dont les sujets doivent avoir commerce avec ces objets selon leur position. Le rapport personnel aux objets d'un individu émerge alors de la pluralité des rapports institutionnels auxquels il a été assujetti. Par exemple, le rapport personnel aux mathématiques d'un professeur s'est forgé à partir des rapports institutionnels aux mathématiques qu'entretiennent la société, l'école, l'université, mais aussi les classes dans lesquelles il était élève, le club d'astronomie ou de dessin auquel il a appartenu, sa famille, etc.

Pour rendre compte de ce que font les personnes au sein des institutions, la TAD postule que toute activité humaine peut se modéliser en termes de praxéologie $[T / \tau / \theta / \Theta]$. La composante praxis $[T / \tau]$ décrit les techniques $\tau$ permettant d'accomplir certains types de tâches T. Alors que dans la composante logos $[\theta / \Theta]$ les technologies $\theta$ - qui sont les discours sur la technique - visent à décrire, expliquer, légitimer, produire les techniques mises en œuvre; ces technologies s'inscrivant elles-mêmes dans des théories $\Theta$ qui les légitiment (Chevallard, 1999). Notre objet d'étude portant sur les conditions et contraintes d'une intégration des pratiques de modélisation mathématique en classe pour résoudre des problèmes issus du réel, nous allons à présent nous attacher à expliciter de quoi sont faites ces praxéologies.

\section{Modéliser en classe de mathématiques}

Chevallard (1989) propose une description en trois étapes du processus de modélisation articulant un système - mathématique ou non - et un modèle (mathématique) de celui-ci:

«1. On définit le système que l'on entend étudier, en en précisant les " aspects" pertinents par rapport à l'étude que l'on veut faire de ce système, soit l'ensemble des variables par lesquelles on le découpe dans le domaine de réalité où il nous apparaît. Nous désignerons ces variables par les lettres $x, y, z, a, b, c$, etc., nous réservant de revenir sur la question - majeure - que soulève cet usage un peu plus loin.

2. On construit alors le modèle à proprement parler en établissant un certain nombre de relations, IR, IR', IR", etc., entre les variables prises en compte dans la première étape, le modèle du système à étudier étant l'ensemble de ces relations.

3. On "travaille" le modèle ainsi obtenu, dans le but de produire des connaissances relatives au système étudié, connaissances qui prennent la forme de nouvelles relations entre les variables du système.

Létape 3 est toujours une phase proprement mathématique, tandis que les étapes antérieures sont du ressort du domaine de réalité dont est censé relever le système - les mathématiques s'il s'agit d'un objet mathématique, etc. » (Chevallard, 1989, p. 53).

Précisons qu'en dépit d'une présentation linéaire en trois étapes, le processus est circulaire car le travail sur le modèle peut conduire à redéfinir le système étudié et ainsi le modèle lui-même. Au regard de ce que propose Chevallard (1989), nous considérerons alors pour notre étude que l'analyse des praxéologies de modélisation nécessite la mise à jour des praxéologies permettant la définition du système, la construction et le travail sur le modèle. La modélisation ne se limite donc pas à un travail 
sur un modèle préconstruit mais appelle l'élaboration d'un discours qui rende compte des ingrédients qui entrent dans l'élaboration du modèle. Ainsi, le rôle et la place des discours qui décrivent, justifient et légitiment les techniques de modélisation apparaissent comme essentiels. C'est pourquoi nous allons établir à présent une typologie des praxéologies qui se fonde sur le rôle et la place du discours technologique.

\section{Des techniques aux praxéologies}

Assude, Mercier, et Sensevy (2007) proposent de classer les techniques mises en œuvre suivant les rapports que les élèves entretiennent avec celles-ci:

\footnotetext{
«Dans le cas des techniques invisibles, l'élève produit une réponse et il est dans un rapport d'action; dans le cas des techniques faibles, l'élève est non seulement dans un rapport d'action (il produit une réponse) mais aussi dans un rapport de formulation (la technique consiste aussi en la manipulation d'outils symboliques et langagiers normalisés, des représentations au sens de Brousseau, 2004); dans le cas des techniques fortes l'élève est dans un rapport d'action (il produit), dans un rapport de formulation (il manipule des représentations et il justifie aussi cette technique - il formule un discours sur la pertinence des représentations utilisées, qui sont des systèmes de notations et les notions associées) et éventuellement dans un rapport de validation (au cas où la justification devient travail de la consistance d'une théorie).»(Assude, Mercier, \& Sensevy, 2007, p. 227).
}

Parce que cette classification des techniques est dialectique avec l'existence, la place et le rôle des éléments technologico-théoriques associés, nous préférons parler de praxéologies - plutôt que de techniques - muettes ${ }^{1}$, faibles ou fortes. Ainsi, nous considérons qu'une praxéologie est muette lorsqu'elle se donne à voir uniquement à travers sa composante praxis; seule la technique mise en œuvre dans un rapport d'action est visible. Nous utilisons le qualificatif « muet» car le fait que seule la technique soit perceptible n'est pas antinomique avec l'existence d'un logos mais qui serait alors tu. En revanche, une praxéologie faible laisse entrevoir la composante logos au travers des ostensifs associés à la technique mise en œuvre mais le discours technologique reste limité à la seule description de la technique dans un rapport de formulation non encore abouti. Enfin, une praxéologie forte met en œuvre dialectiquement les deux composantes praxis et logos dans des rapports d'action, de formulation et de validation.

Ainsi, par exemple, concernant les praxéologies de modélisation, si seul le travail sur un modèle est réalisé sans que ne soient énoncées les hypothèses fondatrices, nous parlerons de praxéologies muettes. En revanche, si les hypothèses sont explicitement formulées sans toutefois que leurs domaines de validité ne soient discutés nous qualifierons ces praxéologies de faibles. Pour pouvoir caractériser une praxéologie de modélisation comme forte, il faudra que soient présentes les trois étapes du processus (définition du système; construction du modèle; le travail sur le modèle) à travers un discours qui explicite leurs rôles et valide le processus lui-même.

Nous allons maintenant présenter le problème que les élèves ont étudié sous la direction de leur professeur.

\section{Analyse a priori du problème à étudier}

\section{Quand la taille du géant devient un problème}

Le problème posé à des élèves de CM2 en mai 2010 porte sur la détermination de la mesure d'une grandeur inaccessible, c'est-à-dire sans pouvoir recourir à un instrument de mesure, ni au report d'un étalon sur l'objet lui-même (ou sa représentation). L'étude à conduire est présentée de façon naive à partir d'une photo et reprend une interrogation familière à propos de la hauteur d'un édifice. La figure 1 est une réduction de l'énoncé tel qu'il était présenté aux élèves sur une feuille A4 où la photo ${ }^{2}$ mesurait $16,1 \mathrm{~cm}$ horizontalement et $12 \mathrm{~cm}$ verticalement.

Au-delà de l'activité de modélisation requise pour répondre à la question posée, ce problème présente plusieurs éléments de rupture avec les types de problèmes habituellement proposés en classe de mathématiques à l'école élémentaire. La réponse à la question posée repose sur des calculs alors qu'aucun nombre sur lesquels ces calculs doivent être réalisés n'est fourni. C'est là une rupture du contrat didactique classique qui prévaut en classe de mathématiques car les élèves doivent trouver un moyen de produire ces nombres. 


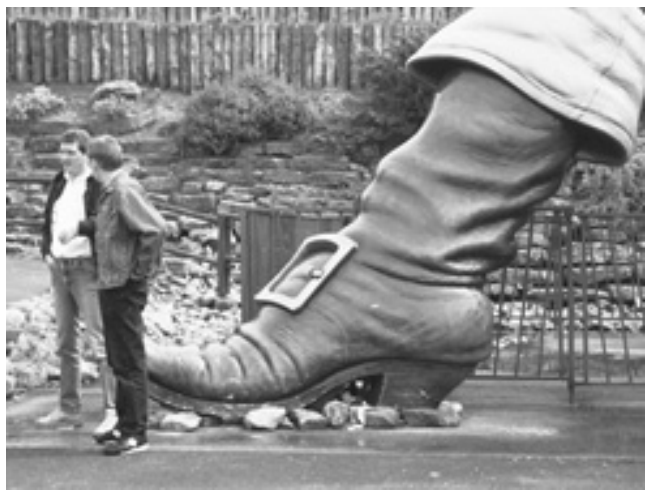

Cette photo a été prise dans un parc d'attraction en Angleterre. On y aperçoit une partie de la jambe d'un géant. Quelle est à peu près la taille de ce géant?

Figure 1. Problème du géant

Au-delà de l'activité de modélisation requise pour répondre à la question posée, ce problème présente plusieurs éléments de rupture avec les types de problèmes habituellement proposés en classe de mathématiques à l'école élémentaire. La réponse à la question posée repose sur des calculs alors qu'aucun nombre sur lesquels ces calculs doivent être réalisés n'est fourni. C'est là une rupture du contrat didactique classique qui prévaut en classe de mathématiques car les élèves doivent trouver un moyen de produire ces nombres.

En second lieu, la locution adverbiale «à peu près » indique d'emblée que la réponse attendue sera nécessairement approximative. En effet, le problème que nous avons proposé à l'étude conduit à travailler sur un modèle de proportionnalité qui nécessite le recours à des grandeurs dont certaines sont des caractéristiques physiques des hommes. Ceci devrait conduire à différencier la taille d'un homme (sur la photo) de la taille des hommes (en général) ou à distinguer les proportions des caractéristiques physiques d'un homme sur la photo, d'un élève de la classe ou de leur professeur. Or pour Brousseau (2005), les besoins de désignation propres à la prise en charge de la variabilité constituent une possible source d'obstacle épistémologique:

« Nous trouvons aussi une difficulté liée à la dualité des moyens linguistiques de la détermination des objets et des classes: par des noms propres ou par des propriétés, par des listes de noms ou par des propriétés d'ensembles, etc. Cette dualité de langage établit une sorte d'équivalence linguistique et "logique" entre un élément, un élément générique et la classe qu'il parcourt. Les mêmes mots désignent les objets et les classes, les fonctions et leurs images etc. L'abus, dénoncé mais fermement maintenu en mathématiques, est partout assez anodin sauf quand le passage de l'ensemble à son représentant ou l'inverse sont problématiques. L'usage sans précaution de la formulation logique classique dans les situations statistiques sera une source permanente d'illusions et d'erreurs pour les débutants, et peut être un obstacle. » (Brousseau, 2005, p. 183).

Nous ne développerons pas davantage cette délicate question ici car nous y reviendrons au cours de l'analyse de la séance observée dans laquelle nous trouverons des traces de ce phénomène.

Enfin, indépendamment du fait qu'il est demandé une valeur approchée de la taille du géant, la validation de la réponse produite par les élèves ne va pas de soi car «la "solution n'est pas connue. Le modèle construit pour produire «une » réponse à la question posée ne pourra pas être validé par un milieu matériel objectif. Les élèves (et leur professeur) doivent ainsi accepter l'incertitude du résultat numérique produit. La validation de la réponse ne pouvant porter que sur le processus de construction de la réponse, c'est-à-dire le processus de modélisation qui permettra alors de déterminer un intervalle d'acceptabilité de la réponse.

Les moments de rupture, en bousculant le régime stationnaire des éléments des systèmes didactiques, donnent à voir certaines de ses caractéristiques. C'est donc bien l'aspect atypique de ce problème qui suscite l'intérêt du chercheur dans ce qu'il révèle des phénomènes didactiques. Le problème présenté, nous allons à présent expliciter la fonction d'une analyse a priori pour notre étude.

\section{Fonction de l'analyse a priori}

Notre objet d'étude portant sur la mise en œuvre $\mathrm{du}$ processus de modélisation, notre analyse se limitera aux praxéologies mathématiques. Pour ce faire, nous devons expliciter le modèle épistémologique de référence que nous utilisons comme grille 
de lecture au travers de laquelle l'observation est interprétée. C'est l'analyse a priori de l'organisation mathématique qui le dévoile. Or toute observation est située dans le temps et dans l'espace. Autrement dit la légitimité des praxéologies (mathématiques) dépend du moment et de l'institution dans laquelle elles sont mises en œuvre, c'est-à-dire du rapport institutionnel à ces praxéologies. Les techniques envisageables pour résoudre un problème se déterminent donc en fonction du rapport institutionnel aux savoirs qui prévaut dans l'institution considérée. Dans notre cas, ce rapport est décrit, a minima, par le programme scolaire en vigueur dans une classe de CM2 en France en mai 2010. Or le programme concernant la proportionnalité est plutôt laconique quant aux techniques à mettre en œuvre:

« La proportionnalité est abordée à partir des situations faisant intervenir les notions de pourcentage, d'échelle, de conversion, d'agrandissement ou de réduction de figures. Pour cela, plusieurs procédures (en particulier celle dite de la "règle de trois") sont utilisées. » (Ministère de l'Éducation Nationale, 2008, p. 23).

Mais qu'elles peuvent être ses différentes «procédures »? Supposons par exemple que le géant est proportionnel à un des hommes de la photo. Nous pouvons calculer la taille du géant comme « $4^{\mathrm{e}}$ proportionnelle » connaissant trois valeurs numériques (la longueur du pied et la taille de l'homme choisi sur la photo, la longueur du pied du géant). Il est alors possible de calculer puis utiliser le coefficient d'agrandissement; recourir à la technique dite du « produit en croix » dans un tableau de proportionnalité; chercher combien de fois la taille de l'homme est plus grande que la longueur de son pied, puis appliquer ce rapport au géant, etc. Indépendamment des différentes manières de calculer la taille du géant que nous venons de décrire, toutes ces techniques particulières reposent sur la même hypothèse et le même modèle de proportionnalité, elles sont donc du même type. Ainsi dans notre analyse a priori nous identifierons des types de techniques comme représentants génériques d'un ensemble de techniques qui se justifient toutes par un même discours technologique.

Si les formulations utilisées dans notre analyse a priori pour énoncer les hypothèses, décrire les techniques ou expliciter les discours technologiques asso- ciés ne sont pas attendues des élèves telles quelles, les praxéologies qu'elles décrivent doivent pouvoir vivre dans la classe. Cela signifie que les élèves auront à mettre en œuvre des techniques du même type que celles qui sont présentes dans notre analyse et que si un «discours sur la technique » est sollicité par le professeur, l'élève regardé comme sujet de l'institution considérée devra produire des discours du même type que ceux que nous allons identifier, dès lors qu'il aura un rapport idoine aux savoirs en jeu. Enfin, la fonction de l'analyse a priori ici étant d'expliciter notre modèle épistémologique de référence, il n'est pas nécessaire d'anticiper les erreurs éventuelles des élèves ou leurs difficultés, contrairement à ce qui peut se faire dans d'autres types d'analyse a priori. En revanche, la mise à jour des techniques qui peuvent vivre nous permettra de reconnaître celles qui ont effectivement vécues.

\section{Les hypothèses sur lesquelles se fondent les techniques}

La question que les élèves ont à étudier conduit à un problème où deux modèles coexistent: un modèle interprétatif des informations fournies par la représentation photographique, un modèle de traitement des informations extrapolées notamment à partir de la représentation. Ces deux modèles sont des modèles de proportionnalité. Les hypothèses faites sont au fondement des modèles de proportionnalité utilisés, et consubstantiellement, les modèles de proportionnalité sont validés par la validation des hypothèses qui les fondent. Tout modèle se fonde sur des hypothèses dont l'énonciation claire et explicite contribue à établir le domaine de validité.

La première hypothèse $(\mathrm{H} 0)$ concerne la photographie: elle est une reproduction fidèle de la réalité, c'est-à-dire sans déformation. Cette hypothèse est fondatrice du modèle de proportionnalité P0 selon lequel les proportions des objets et des personnes sur la photo sont conservées par rapport à la réalité.

P0: Proportionnalité des dimensions sur la photo par rapport aux dimensions réelles.

Une seconde hypothèse ( $\mathrm{Hl}$ ) concerne la conservation des proportions d'un corps d'un être humain à un autre. Ceci signifie que si on choisit une partie du corps pour étalon et si on reporte $n$ fois cet étalon 
dans la taille d'un homme, par exemple, on pourra le reporter $n$ fois dans la taille d'un autre homme. Pour le dire autrement, le corps d'un être humain est toujours l'agrandissement homothétique ${ }^{3}$ d'un autre être humain. Cette hypothèse fonde le modèle de proportionnalité P1.

P1: Proportionnalité des dimensions entre deux êtres humains.

Une troisième hypothèse (H2) concerne le corps du géant: il est un agrandissement homothétique du corps d'un être humain, c'est-à-dire que les dimensions du géant sont proportionnelles aux dimensions d'un être humain. Pour le dire autrement, il y a conservation des proportions du corps du géant et du corps d'un être humain, c'est le modèle de proportionnalité P2.

P2: Proportionnalité des dimensions du géant avec celles d'un être humain.

Ces deux dernières hypothèses appellent un commentaire. Nous avons déjà évoqué la question de la variabilité, notamment les besoins de désignation comme possible source d'obstacle épistémologique (Brousseau, 2005). Il est en effet nécessaire, lorsqu'on traite de variabilité de spécifier le caractère étudié - la grandeur - dont on souhaite connaître la distribution des fréquences; la population sur laquelle la collecte est réalisée et l'élément générique qui représente cette population. Or la mesure des grandeurs associées aux caractéristiques physiques d'un individu, varie d'un individu à l'autre au sein d'une population déterminée (par exemple, les hommes de type caucasien entre trente et cinquante ans). Il n'y a donc pas de réponse unique sauf à considérer un individu particulier. Parler d'un homme comme élément générique d'une population donnée n'est pas équivalent à parler d'un homme en particulier. Ainsi, formellement, les deux hypothèses $\mathrm{H} 1$ et $\mathrm{H} 2$ devraient être spécifiées a minima en évoquant plutôt qu'un être humain, un homme sur la photo, un homme adulte ou un enfant. Un tel niveau de spécification reste cependant faible car statistiquement, il faudrait être plus précis dans la définition des populations concernées. Un enfant de trois ans, un préadolescent ou un adolescent pubère n'ont pas les mêmes morphologies, par exemple. Concrètement, ces questions pour être prises en charge dans la classe devraient faire l'objet d'une étude spécifique conduisant ainsi à réaliser une " enquête » sur les proportions du corps humain.

Sur la base des hypothèses que nous venons d'énoncer, explicitons à présent de quoi sont faites les techniques pour résoudre le problème.

\section{Les types de techniques}

Chacun des modèles de proportionnalité P0, P1, P2 que nous venons de citer peuvent être mis en œuvre sous la forme de la conservation des proportions ou du calcul du coefficient de proportionnalité. La conservation des proportions signifie, par exemple, que si la mesure d'une grandeur A est $n$ fois plus grande que la mesure d'une grandeur $B$ sur la photo, on retrouve la même proportion dans la réalité. En revanche, le recours au coefficient de proportionnalité correspond, par exemple, à l'échelle de la photo ou au rapport d'agrandissement entre un homme de la photo et le géant.

Une observation de la photo conduit à identifier différentes grandeurs qui pourront être aisément mobilisées dans ces modèles mathématiques:

$\mathrm{T}_{\mathrm{G}}$ et $\mathrm{T}_{\mathrm{H}}$ : la taille du géant ou d'un homme.

$h_{G}$ et $h_{H}$ : la hauteur du pied du géant ou celle d'un homme.

$\mathrm{L}_{\mathrm{G}}$ et $\mathrm{L}_{\mathrm{H}}$ : la longueur du pied ${ }^{4}$ du géant ou celle d'un homme.

$\mathrm{S}_{\mathrm{G}}$ et $\mathrm{S}_{\mathrm{H}}$ : la longueur de la semelle de la botte du géant ou de la chaussure d'un homme.

$\mathrm{b}_{\mathrm{G}}$ : la hauteur de la botte du géant.

Par ailleurs, trois domaines de réalité coexistent: la photo; une réalité imaginée, supposée, évoquée; la réalité vécue par l'élève dans ou hors de la classe. Ainsi, pour déterminer la mesure d'une grandeur identifiée sur la photo, par exemple la longueur de la semelle d'une chaussure, il est possible de mesurer cette longueur sur la photo en considérant un des deux hommes. Mais l'élève peut aussi imaginer ce que pourrait être cette mesure, l'extrapoler à partir de la mesure de sa chaussure ou encore mesurer la 
chaussure de son professeur. Ainsi, à chaque grandeur considérée pourra être associée une mesure dans un de ces trois domaines de réalité.

Afin de distinguer ces différents domaines de réalité nous utilisons les notations ci-dessus $\left(\mathrm{T}_{G}, \mathrm{~T}_{\mathrm{H}}\right.$, $h_{G}, h_{H}, L_{G}, L_{H}, S_{G}, S_{H}, b_{G}$ ) pour désigner une valeur arbitrairement attribuée à la mesure des grandeurs correspondantes dans la réalité évoquée par la photo. Lorsque ces mêmes grandeurs sont mesurées sur la photo nous ajoutons le préfixe $\mu: \mu \mathrm{T}_{\mathrm{H}}, \mu \mathrm{h}_{\mathrm{G}}, \mu \mathrm{h}_{\mathrm{H}}$, $\mu L_{G}, \mu L_{H}, \mu S_{G}, \mu S_{H}$. Enfin, lorsque les grandeurs sont mesurées dans la réalité vécue par l'élève nous ajoutons au préfixe $\mu$ l'astérisque ${ }^{*}: \mu \mathrm{T}_{\mathrm{H}}^{*}, \mu \mathrm{h}^{*}{ }_{\mathrm{H}}, \mu \mathrm{L}^{*}{ }_{\mathrm{H}}$, $\mu \mathrm{S}^{*}{ }_{\mathrm{H}}, \mu \mathrm{b}^{*}{ }_{\mathrm{G}}$ (dans ce dernier cas, c'est la hauteur d'une botte quelconque qui est mesurée). Ces notations rendent compte des techniques présentes en explicitant les domaines de réalité concernés.

Sous l'hypothèse Ho, trois types de techniques sont envisageables à ce niveau-là de la scolarité, nous les noterons dans la suite $\tau_{1}, \tau_{2}$ et $\tau_{3}$.

Le premier type de technique consiste à choisir comme étalon un élément commun au corps du géant et à celui d'un des hommes de la photo, puis à déterminer le nombre de reports de cet étalon dans le corps d'un homme adulte sur la photo. En supposant que le géant est un agrandissement homothétique d'un des hommes de la photo, la taille du géant s'obtient en multipliant ce rapport par la dimension réelle supposée de l'étalon. Par exemple, si on considère que la hauteur base du pied-base du mollet peut être reportée sept fois dans le corps d'un homme et si on considère, d'après la photo, que cette hauteur pour le géant est égale à la taille d'un homme, alors la taille du géant est sept fois la taille ${ }^{5}$ d'un homme - par exemple 1,75 m - soit 12,25 m (la précision au $\mathrm{cm}$ près est assez dérisoire ici).

$$
\begin{gathered}
\tau_{1}: \mathrm{T}_{\mathrm{G}}=\mathrm{k}_{1} \times \mathrm{h}_{\mathrm{G}} \text { où } \mathrm{k}_{1}=\mu \mathrm{T}_{\mathrm{H}}^{*} / \mathrm{hh}^{*}{ }_{\mathrm{H}}=7 \text { (report) et } \\
\mathrm{h}_{\mathrm{G}}=\mathrm{T}_{\mathrm{H}}=1,75 \mathrm{~m} \text { (moyenne, INSEE) }
\end{gathered}
$$

Le second type de technique consiste à appliquer le coefficient d'agrandissement entre un homme sur la photo et le géant. Ainsi par exemple, en mesurant la longueur des semelles des chaussures d'un homme et du géant sur la photo, on obtient respectivement $1,2 \mathrm{~cm}$ et $9 \mathrm{~cm}$, ce qui donne un coefficient d'agrandissement de 7,5. Si on choisit (arbitrairement) de prendre 1,80 m comme taille d'un homme, celle du géant est alors 7,5 fois plus grande soit une taille de $13,5 \mathrm{~m}$.

$$
\begin{aligned}
& \tau_{2}: \mathrm{T}_{\mathrm{G}}=\mathrm{k}_{2} \times \mathrm{T}_{\mathrm{H}} \text { où } \mathrm{k}_{2}=\mu \mathrm{S}_{\mathrm{G}} / \mu \mathrm{S}_{\mathrm{H}}=9 / 1,2 \text { et } \mathrm{T}_{\mathrm{H}} \\
= & 1,80 \mathrm{~m} \text { (estimation) }
\end{aligned}
$$

Enfin, le troisième type de technique, dont nous pouvons déjà dire qu'il n'est pas apparu dans la classe observée ici mais dans d'autres classes de notre corpus, est la composée des deux types de techniques précédents. Nous le décrivons à partir d'un exemple. En supposant que les proportions du géant sont les mêmes que celle d'un homme et en estimant que la longueur du pied d'un homme de $1,80 \mathrm{~m}$ mesure $30 \mathrm{~cm}$, alors la taille du géant est égale à 6 fois $(180: 30=6)$ la longueur de son propre pied. Reste à déterminer la longueur du pied du géant, ce qui peut se faire en appliquant le coefficient d'agrandissement entre un homme sur la photo et le géant. En mesurant sur la photo les longueurs des semelles des chaussures du géant et d'un homme, on trouve donc que la longueur du pied du géant est 7,5 fois $(9: 1,2=7,5)$ plus grande que celle de l'homme choisi sur la photo. Ainsi, en supposant que la longueur du pied de l'homme mesure $30 \mathrm{~cm}$, celle du pied du géant mesurera $7,5 \times 0,30 \mathrm{~m}=2,25 \mathrm{~m}$ et sa taille sera de $6 \times 2,25 \mathrm{~m}=13,50 \mathrm{~m}$.

$$
\begin{aligned}
\tau_{3}: T_{G} & =k_{1} \times S_{G}=k_{1} \times\left(k_{2} \times S_{H}\right) \text { où } k_{1}=T_{H} / S_{H} \\
=180 / 30 & =6(\text { estimation }) \text { et } k_{2}=\mu S_{G} / \mu S_{H}=9 / 1,2
\end{aligned}
$$
$=7,5$

Les trois types de techniques que nous venons de décrire utilisent l'hypothèse $\mathrm{H} 2$ selon laquelle le géant est un agrandissement d'un homme ce qui permet de déterminer la mesure d'une grandeur sur le géant à partir de celle correspondante sur un homme de la photo. Dans le cas de $\tau_{1}$ le report dans la réalité de la hauteur base du pied-base du mollet utilise l'hypothèse $\mathrm{Hl}$ selon laquelle les dimensions entre deux hommes sont proportionnelles, ce qui n'est pas le cas pour $\tau_{2}$ où les mesures sont faites directement sur la photo.

Notons, enfin, que le géant porte des bottes avec un talon, il y a donc une différence entre la taille du géant (nus pieds) et sa hauteur (comme édifice).

L'analyse a priori des praxéologies mathématiques que nous venons de réaliser montre que trois modèles de proportionnalité peuvent être mobi- 
lisés et qu'indépendamment de ces modèles, trois domaines de réalité coexistent et trois types de techniques peuvent être mis en œuvre. Ainsi, ces différentes praxéologies ne produisent pas seulement des réponses différentes mais des " constructions » différentes du problème. Dans tous les cas la proportionnalité est utilisée comme cadre de modélisation mais des organisations mathématiques différentes sont construites suivant les hypothèses et les types de techniques mis en œuvre. Ce phénomène didactique a été repéré par Orange (2005) qui parle alors de problématisation. C'est cette question que nous allons aborder à présent pour éprouver la robustesse de notre propre cadre théorique.

\section{La problématisation}

À partir de trois exemples ${ }^{6}$ fort différents - le problème pratique d'une voiture qui démarre mal; l'explicitation par des élèves de cycle 3 de l'école élémentaire des relations entre la nutrition et la «force » des différentes parties du corps; le jeu de la tour de Hanoï - Orange (2005) illustre comment les mises en ouvre de praxéologies différentes pour résoudre un problème traduisent des constructions différentes du problème:

« Devant la même tâche, qui leur fait problème, c'est-à-dire pour laquelle elles n'ont pas de réponse immédiate, deux personnes pourront ainsi avoir construit le problème de façon différente. Cette construction, liée aux projets qu'elles se donnent et aux connaissances qu'elles ont déjà, les conduira à l'élaboration d'une connaissance qui ne sera pas du tout la même; alors que pour un observateur leurs solutions peuvent paraître proches. » (Orange, 2005, p. 73).

L'analyse réalisée précédemment illustre, nous semble-t-il ce phénomène didactique. Dans tous les cas la proportionnalité est mobilisée comme cadre de modélisation mais des organisations mathématiques différentes sont construites suivant les hypothèses et les types de techniques mis en œuvre. Cependant, le concept de problématisation de Orange ne se limite pas à « une façon de penser les relations entre problèmes et solutions » (Ibid, p. 69), c'est-à-dire ici entre système et travail du modèle. Il se définit comme « la construction explicite d'un champ de possibles » (Ibid, p. 70), intégrant la part de construction du modèle dans le processus de modélisation:

«[...] la dynamique des problèmes ne se fait pas simplement par conjectures et réfutations; il y a développement et transformation de problématiques, par la mise en jeu des deux types de questions pointées plus haut (comment est-ce possible? Pourrait-il en être autrement?), dans un cadre théorique qui bouge. C'est ce travail de la pensée scientifique (toujours au sens large) qui confère des caractéristiques spécifiques aux problématisations développées: il ne s'agit pas simplement de construire un problème pour produire une solution, mais d'explorer et de « cartographier » le champ des possibles. Cette exploration a pour résultat un caractère essentiel des savoirs scientifiques: leur « nécessité » (apodicticité). » (Ibid, p. 78).

Le simple recours impensé à la proportionnalité comme technique permettant de résoudre un problème ne peut être considéré comme une activité de modélisation. La part du discours technologique des praxéologies de construction du modèle, parait donc essentielle dans le processus de validation du modèle ainsi construit. Or pour construire une réponse à une question, la classe convoque un certain milieu fait de différentes ressources. Parmi ces ressources, certaines sont en quelque sorte des réponses « toutes faites » qui ont été validées par telle institution qui lui confère son « estampille ». D'autres ressources sont des outils d'analyse qui permettent d'interroger et évaluer ces réponses partielles à la question génératrice de l'étude et fournissent des matériaux à partir desquels la réponse de la classe est produite. Chevallard (2011) appelle ce processus la dialectique des médias et des milieux. Notons que pour Chevallard est média tout système producteur de messages à l'adresse d'un public, quel qu'il soit: une personne, un quotidien, un ouvrage ou une émission de radio sont des médias. Il résume alors le travail d'un système didactique $S(X ; Y ; Q)$ formé autour d'une question $Q$ par une classe $X$ et le professeur $Y$ qui en dirige l'étude par le schéma herbartien:

$$
M=\left\{R_{1}^{\diamond}, R_{2}^{\diamond}, \ldots, R_{n}^{\diamond}, O_{n+1}, \ldots, O_{m}\right\}
$$

dans lequel le milieu $M=\left\{R_{1}^{\diamond}, R_{2}^{\diamond}, \ldots, R_{n}^{\diamond}, O_{n+1}, \ldots, O_{m}\right\}$ fait de réponses institutionnellement estampillées $R_{i}^{\diamond}$ et d'œuvres de la culture $O$ doit permettre la construction de la réponse $R \cdot$ de la classe. 
Supposons qu'un élève déclare qu'un homme a les mêmes proportions qu'un enfant. Quel crédit accordé à la réponse estampillée par cet élève? Quel milieu construire pour mettre à l'épreuve une telle assertion? Dans la classe que nous considérons dans ce texte, rien n'est construit pour ce faire, c'est ce qui nous conduira à qualifier de faible le milieu dans lequel elle évolue. Mais dans une des classes de notre corpus un professeur a spontanément proposé de faire une enquête en faisant comparer des rapports de longueurs sur des adultes (les parents des élèves) et des enfants (les élèves eux-mêmes). La mise en cuvre d'une dialectique des médias et des milieux permet donc in fine d'estimer le degré d'incertitude d'une assertion. Un tel procédé nourrit ainsi une pédagogie de l'enquête (Ladage \& Chevallard, 2011).

Il nous semble alors que ce que Orange nomme la saturation du registre empirique ou du registre des modèles pourrait correspondre à la mise en œuvre d'une dialectique des médias et des milieux:

« Nous parlons de saturation du registre empirique lorsque le maître ou les élèves introduisent de nouvelles observations, expériences ou documents relatifs à des observations ou expériences. Lorsque ce sont des documents sur des modèles (documentaires par exemple) qui sont introduits, c'est le registre des modèles qui est saturé. » (Orange, 2007, note 14, p. 54).

Dans le cas du problème étudié, une validation empirique de la solution élaborée parait difficile: professeur et élèves ne savent pas dans quel parc d'attraction se trouve ce géant dont on cherche à connaître la taille. Si une enquête avec l'Internet est toujours envisageable, ce n'est pas le choix qui est fait. En ce cas, comment établir le domaine de validité de la réponse produite? Une telle validation passe alors nécessairement par la validation du processus de production de la réponse via la confrontation à un milieu «théorique ». Nous rejoignons ici le point de vue de Orange (2007):

Lorsqu' « il n'y a pas possibilité de validation par l'élève de ses modèles et de ses argumentations grâce à un milieu objectif antagoniste. Se développe ainsi un espace problématique qui permet l'exploration et la délimitation du champ des possibles, par établissement de conditions de possibilité des solutions (nécessités). Ce champ sera secondairement peuplé par des solutions validées par saturation du registre empirique ou du registre des modèles ${ }^{7}$. Si le premier mode correspond bien à un apprentissage par adaptation (au moins dans ses premières phases), le second fonctionne par pratique théorique, c'est-à-dire par construction de solutions possibles et examen critique de ces solutions. » (Orange, 2007, p. 46).

Il apparait ainsi qu'il ne peut y avoir construction de modèles au sens du processus de modélisation décrit par Chevallard, sans la mise en œuvre d'une dialectique des médias et des milieux qui permette une explicitation de la problématisation. Si les hypothèses qui fondent le modèle ne sont pas énoncées ou si elles le sont sans être interrogées ni légitimées, sans que leur domaine de validité ne soit exploré, alors nous considérerons que le processus de modélisation ne s'est pas pleinement développé et l'étape de construction du modèle partiellement réalisée. Notre analyse devra alors répondre à la question : existe-t-il dans la classe observée des praxéologies qui donnent du sens à ce qui est fait ou l'activité des élèves se limite-t-elle à une simple application de la proportionnalité? Autrement dit, les élèves perçoivent-ils que la proportionnalité est utilisée comme outil de modélisation d'une situation? Ces questions qui sont au cœur de notre étude reprennent en réalité une distinction ${ }^{8}$ fondatrice de la didactique des mathématiques que Sarrazy (2007) formule en ces termes: « rapporter la question du sens des connaissances à leurs usages via les situations vs produire des situations permettant l'usage des connaissances pour en "montrer" le sens. » (Sarrazy, 2007, p. 32).

Notre cadre théorique exposé, nous explicitons à présent notre méthodologie de recherche.

\section{Méthodologie de la recherche}

\section{Recueil des donnéeses}

L'observation dont nous allons rendre compte a été réalisée par nous-même dans le cadre d'une étude exploratoire du groupe ACODIS ${ }^{9}$ de l'IUFM d'Alsace visant à identifier les variables liées à l'enseignement susceptibles d'avoir un effet sur les apprentissages des élèves. Le protocole suivi devait donc permettre d'observer les praxéologies mathématiques spontanées des six professeurs volontaires, c'est-à-dire leurs pratiques 
ordinaires lorsqu'ils enseignent les mathématiques, ce qu'ils font et disent naturellement, de façon habituelle. Ainsi, il était essentiel qu'aucune indication quant à la façon de conduire leur enseignement ne soit donnée. Dans la mesure où il s'agissait d'une première étude de type exploratoire nous ne nous sommes pas attachés à définir des critères de représentativité autre que la diversité des types d'écoles concernées (écoles rurale, en périphérie d'une grande ville, dans une ville de taille moyenne ou dans un village, en zone prioritaire d'une grande ville). Les professeurs qui ont accepté de participer à cette recherche n'ont pas contribué à l'élaboration du protocole ou à l'analyse des données recueillies. L'étude de cas dont nous rendons compte dans ce texte porte sur une classe de CM2 de vingt-cinq élèves considérée comme ayant un bon niveau scolaire par leur professeur qui est une femme ayant moins de cinq ans d'ancienneté dans le métier. Nous emploierons le masculin pour dépersonnaliser l'observation.

Après un premier contact visant à préciser les enjeux et les conditions de l'observation, le problème a été envoyé à chaque professeur par courrier électronique. L'étude du problème, dirigée par le professeur selon ses habitudes d'enseignement, a été précédée et suivie par la passation d'un même test, sans temps limité, qui comportait cinq exercices et dont le professeur ne devait pas prendre connaissance. Dans le premier exercice les élèves devaient effectuer des multiplications à trou $(4 \times \ldots=6 ; 4 \times \ldots=7 ; 5 \times$ $\ldots=8$ ). Le second exercice demandait d'estimer la hauteur d'un arbre vu entièrement sur une photographie devant lequel une élève - dont l'énoncé précisait qu'elle était dans une classe de CM2 - passait. Les troisième et quatrième exercices représentaient le dessin d'un pêcheur debout et vu de face avec à ses pieds un poisson dessiné entièrement en position horizontale comme posé au sol. L'énoncé précisait que le pêcheur mesurait 1,80 m et il fallait estimer la taille du poisson. Le cinquième et dernier exercice représentait le dessin simplifié d'un voilier sur lequel était indiquée la hauteur du mât $(6 \mathrm{~cm})$ et la longueur du pont $(9 \mathrm{~cm})$. Il fallait calculer la longueur du pont sachant qu'après un agrandissement la hauteur du mât était de $15 \mathrm{~cm}$.

De son côté, le professeur a répondu à deux questionnaires avant et après la résolution du problème en classe. Ainsi, pendant que les élèves passaient le prétest, le professeur a répondu à la première partie du questionnaire dans lequel il devait fournir sa solution du problème, dire s'il avait déjà donné un problème de ce type et s'il pensait que ses élèves pourraient tirer profit de son étude ou au contraire s'il pourrait être délicat de donner ce problème à étudier. La seconde partie de ce premier questionnaire a été envoyée par messagerie électronique dans les jours qui ont suivi. Les questions portaient alors sur les difficultés supposées des élèves, sur leurs procédures éventuelles, les connaissances ou compétences requises pour résoudre le problème et sur ses particularités. Le professeur avait une semaine pour adresser aux chercheurs sa fiche de préparation de la ou des séances d'enseignement qu'il envisageait de conduire pour l'étude du problème avec sa classe. L'objet de la recherche portant sur les pratiques spontanées des professeurs, cette fiche ne faisait l'objet d'aucun commentaire de la part des chercheurs. Une semaine après cet envoi, l'étude du problème a été filmée dans les classes en caméra fixe, les professeurs portant un micro-cravate. À l'issue des séances d'enseignement, les élèves ont passé le test initial une seconde fois - sans temps limité - et le professeur a répondu à un dernier questionnaire qui visait à cerner son ressenti et l'analyse qu'il faisait de l'expérimentation. Les questions portaient sur les difficultés rencontrées par les élèves ou par lui-même pour mettre en œuvre sa séance; sur les procédures de résolution accessibles aux élèves; les connaissances et/ ou compétences requises pour résoudre le problème; les éléments qui l'avaient surpris et l'enseignement qu'il tirait de cette expérience.

La description du protocole appelle un commentaire concernant le test que les élèves ont passé avant et après l'étude du problème. Il apparait nettement que le contenu de ce test constitue un élément du milieu des élèves dans l'étude du problème du géant. Cependant, la séance d'enseignement a eu lieu plus de quinze jours après la passation du test et les élèves savaient qu'ils ne devaient pas en parler à leur professeur. L'enrichissement du milieu des élèves sans que les professeurs ne sachent de quoi est fait cet enrichissement est une contrainte de la situation du professeur dans ce contexte. Notons, cependant, que le contenu du test n'a jamais été évoqué par les élèves de la classe que nous avons observée.

Les observations ont eu lieu dans la deuxième quinzaine du mois de mai 2010 et la proportionnalité avait été étudiée sans que les chercheurs ne 
connaissent le contenu de cet enseignement. En plus des vidéos réalisées, des tests des élèves, des questionnaires des professeurs et des fiches de préparation, nous avons recueilli les productions écrites des élèves durant l'étude du problème.

\section{Traitement des données et démarche de recherche}

Pour notre analyse, nous nous appuyons sur une transcription de la vidéo complétée par l'enregistrement sonore réalisé par micro-cravate posé sur le professeur. Ceci nous permet de repérer la chronologie de la séance d'enseignement découpée en « scènes » identifiées par les modalités de travail des élèves (collectif, en binôme, individuel) et, pour une même organisation didactique, par le changement d'objet d'étude ou de tâche.

L'analyse a priori vise à déterminer les praxéologies de modélisation qui peuvent vivre, aussi l'absence de telle ou telle de leurs composantes permet d'identifier et de qualifier les praxéologies de modélisation qui ont réellement vécu dans la classe.

Nous nous intéressons aux praxéologies mathématiques mises en œuvre pour l'étude dirigée par un professeur d'un problème, aussi notre analyse se décline-t-elle en quatre temps: tout d'abord la mise à jour des techniques publiques qui ont vécu; l'analyse des discours sur les techniques qui révèle de quoi celles-ci sont faites; l'identification des arguments sur lesquels repose la validation des techniques; le contenu de la correction collective qui expose à la classe les savoirs en jeu.

Nous postulons que la rencontre d'un élève avec un savoir se fait par l'intermédiaire de la classe, c'est pourquoi nous nous intéressons au discours des élèves de façon indifférenciée. Nous ne considérons donc pas ici les élèves dans leur singularité, comme individus isolés dans la classe, mais comme éléments d'un système didactique. Néanmoins, si nécessaire, nous ne nous interdisons pas de consulter les traces écrites d'un élève particulier pour comprendre son intervention dans la classe ou appréhender la pertinence de la réaction du professeur.

Les réponses du professeur observé aux questionnaires permettent d'une part d'identifier son rapport personnel à la modélisation mathématique et d'autre part d'étayer notre analyse relative à ce qui est le véritable enjeu didactique de la séance.

\section{Une étude de cas}

La réception du problème

Avant de regarder comment le professeur observé a conduit l'étude du problème, nous allons examiner, à travers ses réponses aux questionnaires, comment il l'analyse. Pour résoudre ce problème, il utilise une « démarche » qu'il présente en trois étapes et dont il pense qu'elle sera aussi celle des élèves:

$« \rightarrow$ la taille d'un homme adulte $(\approx 1,80 \mathrm{~m})=$ la hauteur du pied du géant

$\rightarrow$ il faut chercher quelle proportion du corps représente la hauteur d'un pied (exemple: il faut 10 hauteurs de pied pour arriver à la taille totale d'un corps humain). Pour cela il faut prendre des mesures sur un « vrai » corps humain

$\rightarrow$ enfin, il reste à multiplier la taille de l'homme adulte par le nombre de hauteurs de pied trouvé en phase (2)

exemple: $1,80 \times 10=$ la taille approximative $\mathrm{du}$ géant ».

C'est donc une technique du type $\tau_{1}$ (report d'un étalon sur un corps) qui est envisagée en recourant de façon implicite à la proportionnalité des dimensions du géant avec celles d'un être humain (modèle P2) ou plus généralement à la proportionnalité des dimensions entre deux êtres humains (modèle P1).

Habituellement, ce professeur propose « des problèmes figurant dans le manuel de mathématiques (pour comprendre les maths, CM2, éd. Hachette éducation). Ces derniers sont toujours en lien avec la notion (la compétence) abordée durant la séance d'apprentissage ». Aussi, il considère que « cette situation de recherche est inédite » car « les élèves n'ont pas l'habitude d'être face à une telle situation de recherche (résolution du problème en plusieurs étapes, stratégies de résolution variées et non induites par la séance d'apprentissage menée précédemment 
en classe, importance de confronter son point de vue à celui des camarades, ...) (+ justifier) ».

Le professeur attribue par ailleurs deux particularités à ce problème, d'une part que « tous les indices, sur lesquels les élèves devront s'appuyer pour résoudre le problème, se trouvent dans la photo et non pas dans l'énoncé. Il s'agit d'indices implicites » et d'autre part, le fait « qu'il est impossible de trouver une réponse exacte à ce problème ». Mais il manque des mots à ce professeur pour rendre compte de la spécificité du problème et ce qui est derrière l'évocation de la variété des stratégies ou l'existence d'indices implicites, est en réalité le processus de modélisation mathématique. Lorsqu'il est amené à détailler « les connaissances et/ou compétences utiles à la résolution du problème », il décline des thèmes d'étude du programme: «L'échelle ( $x \mathrm{~cm}$ sur la photo $=y \mathrm{~cm}$ dans la réalité) ; les grandeurs (conversion d'unités); la technique multiplicative; l'agrandissement; la proportionnalité » témoignant d'un rapport personnel aux mathématiques idoine au rapport institutionnel de son niveau d'enseignement. La modélisation mathématique n'est pas mentionnée dans le programme d'enseignement et la démarche d'investigation n'est présente que dans la part relative à l'enseignement des sciences. Ce professeur dont nous venons de voir qu'il connait bien les programmes de son niveau d'enseignement n'identifie donc pas la modélisation mathématique comme un savoir à mobiliser. Néanmoins il anticipe une difficulté dont il pense qu'elle sera surmontée par une organisation du travail en groupe: « certains élèves risquent d'être impressionnés et "bloqués " face à ce problème inédit dans sa forme. Certains élèves, face à un obstacle de ce type risquent de " baisser les bras ". Pour persévérer dans la recherche j'envisage de les faire travailler en groupe, et de proposer des outils ou des aides adaptés... ». Enfin, si le professeur se demande "Comment les aider sans trop induire une démarche à suivre? », il ne doute pas que $70 \%$ à $90 \%$ de ces élèves réussiront « grâce à la recherche en binôme et à la pause méthodologique notamment ».

En conclusion, nous pouvons anticiper que la modélisation n'ayant pas été identifiée comme un savoir en jeu dans le problème, la rencontre avec le processus de modélisation ne pourra pas être un enjeu didactique dans la séance. C'est ce que nous allons détailler à présent.

\section{Organiser l'étude}

L'étude a été organisée au cours d'une seule (longue) séance d'une durée de 1 h 25 min. Après la distribution d'une feuille avec la photo et l'énoncé, tout débute par une discussion collective de près de cinq minutes amenée par deux questions : "À quelle question devez-vous répondre? De quoi aurez-vous besoin?». Une première recherche en binôme est alors proposée pendant une dizaine de minutes, suivie d'une première mise en commun: «Vous allez juste expliquer votre stratégie, c'est-à-dire comment je m'y suis pris pour démarrer la recherche ». Après dix minutes de discussion collective, un temps de recherche individuel a été proposé structuré par un document sur lequel figuraient quatre consignes:

Ce que je cherche:

Ce que je sais déjà (grâce à la photo, à l'énoncé) :

Ce que je vais faire pour trouver la taille du géant (textes, dessins, calculs, ...) :

Ma proposition de réponse:

Après vingt minutes, cette dernière recherche s'est conclue par la collecte des solutions numériques des élèves notées au tableau et une discussion collective à propos de leur validité pendant vingt minutes encore. La séance s'est achevée par une correction rédigée collectivement et copiée par chaque élève durant vingt minutes.

Ainsi, les durées des temps de recherche (en binôme ou individuel) et de mise en commun collective sont à peu près identiques laissant une part substantielle au discours technologique. Regardons à présent les techniques qui ont vécues publiquement dans la classe.

\section{Un même type de techniques}

Après une première discussion collective et une première recherche en binômes, le professeur sollicite les élèves «Vous allez juste expliquer votre stratégie, c'est-à-dire comment je m'y suis pris pour démarrer la recherche ». C'est ainsi qu'une élève expose maladroitement une technique de type $t_{2}$ 
(application du coefficient d'agrandissement entre un homme sur la photo et le géant). Or le professeur adopte à ce moment-là un strict principe de neutralité quant à la validité des techniques exposées. Il limite donc son intervention auprès de cette élève au fait qu'un simple changement d'unités n'apporte pas d'information nouvelle, sans évaluer la technique elle-même :

E: J'ai pris la taille de la chaussure du géant en centimètres.

$\mathrm{P}$ : Donc sur la photo.

E: J'ai trouvé $9 \mathrm{~cm}$ et après j'ai converti en mètres, ce qui fait $0,09 \mathrm{~m}$ et après on a pris la taille en centimètres du monsieur et on l'a converti en mètres cela fait 0,01 .

$\mathrm{P}$ : Donc tu penses qu'en vrai un homme adulte mesure $0,01 \mathrm{~m}$ ? ça t'a servi à quoi de faire cette conversion?

$\mathrm{E}$ : Ben à convertir les chaussures.

P : Oui mais c'est la même chose en fait $9 \mathrm{~cm}$ c'est pareil que 0,09 m. Est-ce que pour toi c'est en réalité la hauteur de la chaussure du géant en vrai? Elle fait $9 \mathrm{~cm}$ en vrai? Parce que $9 \mathrm{~cm}$ c'est pareil que 0,09. Donc ça t'a servi à quoi de faire la conversion?

E: C'était pour savoir combien elle faisait en mètres.

$\mathrm{P}$ : Et ensuite comment tu t'en es servi de ça?

E: Après on a pris la taille du monsieur en centimètres et on l'a convertie en mètres.

P: Taille de l'adulte sur la photo, combien? [P note au tableau]

E: On a trouvé 0,08 .

$\mathrm{P}$ : Donc $8 \mathrm{~cm}$ que vous avez converti, oui ...

E: Et après on a fait un tableau de proportionnalité et on a cherché... parce qu'en fait on voulait convertir en mètres mais en fait si ça fait pareil ...

P : 9 cm c'est pareil que 0,09 m c'est la même chose; c'est juste exprimé dans une autre unité mais ça représente la même longueur.

$\mathrm{P}$ : Donc après, vous avez essayé de faire un tableau de proportionnalité mais...tu veux aller plus loin ou ... non, je vais juste noter...

On le voit, l'élève n'a pas su exposer sa technique clairement et complètement et la focalisation du professeur sur l'inutilité de la conversion réalisée l'a conduite, par un effet de contrat, à abandonner sa technique qui ne sera plus évoquée par la suite dans la classe, sans même avoir été exposée ni discutée. Or une analyse de la production de cette élève (voir figure 2) confirme qu'elle a utilisé l'échelle $1 \mathrm{~cm}$ sur la photo représente $1 \mathrm{~m}$ en réalité, ce n'était donc pas un problème de conversion. La technique utilisée consiste en effet à supposer d'une part que la photo est une reproduction fidèle de la réalité sans déformation (hypothèse H0) et d'autre part que la hauteur du géant est proportionnelle à la taille d'un homme sur la photo (hypothèse H2). Ce faisant, il est possible de calculer le coefficient d'agrandissement entre un homme de la photo et le géant en calculant le rapport des mesures de longueur des semelles des chaussures $\left(k_{2}=\mu S_{G}\right.$ ' $\left.\mu S_{\mathrm{H}}=0,01 / 0,09=9\right)$. Ce coefficient appliqué à la taille de l'homme mesurée sur la photo $\left(\mathrm{k}_{2} \times \mu \mathrm{T}_{\mathrm{H}}=9\right.$ $\times 0,08=0,72 \mathrm{~m}$ ) permet de déterminer quelle serait la taille du géant sur la photo si celle-ci était assez grande. Il ne reste plus alors qu'à appliquer l'échelle de la photo pour déterminer la taille réelle du géant. Cette technique $\tau_{2 b i s}: T_{G}=e \times\left(k_{2} \times \mu T_{H}\right)$ où $k_{2}=\mu S_{G}$ ' $\mu S_{H}$ est correcte, seule l'échelle choisie $(e=1 / 100)$ est fausse. Or il était possible de calculer approximativement cette échelle en rapportant la taille d'un homme sur la photo à sa taille supposée dans la réalité, par exemple.

Nous faisons l'hypothèse que le professeur ne « reconnait» pas cette technique comme valide et focalise son attention sur l'inutilité de la conversion des mesures prises sur la photo car elle n'est pas du même type $\tau_{1}$ que celle qu'il utilise lui-même et dont il pense qu'elle sera celle des élèves. Le professeur cherche alors moins à résoudre le problème avec les élèves qu'à enseigner sa solution. De fait, seules les techniques du type $\tau_{1}$ - où le nombre de reports d'un étalon dans le corps d'un homme est appliqué au géant - continueront à vivre dans la classe et seront discutées collectivement. Les élèves vont reporter la hauteur d'une botte ou la longueur d'une semelle de chaussure dans un corps d'homme et multiplier ce nombre par la taille de la botte du géant ou la longueur de sa semelle supposées égales à la taille d'un homme sur la photo:

$$
\begin{aligned}
& \tau_{1 \mathrm{a}}: \mathrm{T}_{\mathrm{G}}=\mathrm{k}_{1} \mathrm{~b}_{\mathrm{G}} ; \mathrm{b}_{\mathrm{G}}=\mathrm{T}_{\mathrm{H}} \text { avec } \mathrm{k}_{1}=\mathrm{T}_{\mathrm{H}} / \mathrm{b}_{\mathrm{H}}^{*} \\
& \tau_{1 \mathrm{~b}}: \mathrm{T}_{\mathrm{G}}=\mathrm{k}_{1} \mathrm{~S}_{\mathrm{G}} ; \text { avec } \mathrm{k}_{1}=\left(\mathrm{k}_{1}{ }_{1}+\mathrm{k}_{1}{ }_{1}\right) / 2 \text { et } \mathrm{k}_{1}^{\prime}=\mu \mathrm{T}_{\mathrm{H}^{\prime}}{ }^{\prime} \\
& \mu S^{*}{ }_{H} \text { (report); } k_{1}=\mu T_{H} / \mu S_{H} \\
& \tau_{1 c}: T_{G}=k_{1} b_{G} \text {; avec } b_{G}=\text { "un peu plus que" } T_{H} \text { et } \\
& \mathrm{k}_{\mathrm{l}}=\mathrm{T}_{\mathrm{H}} / \mathrm{b}_{\mathrm{H}} \text { (report supposé) validé par } \\
& \mathrm{k}_{1}=\mu \mathrm{T}_{\mathrm{H}}^{*} / \mu \mathrm{b}_{\mathrm{H}}^{*} \text { (report sur le professeur). }
\end{aligned}
$$




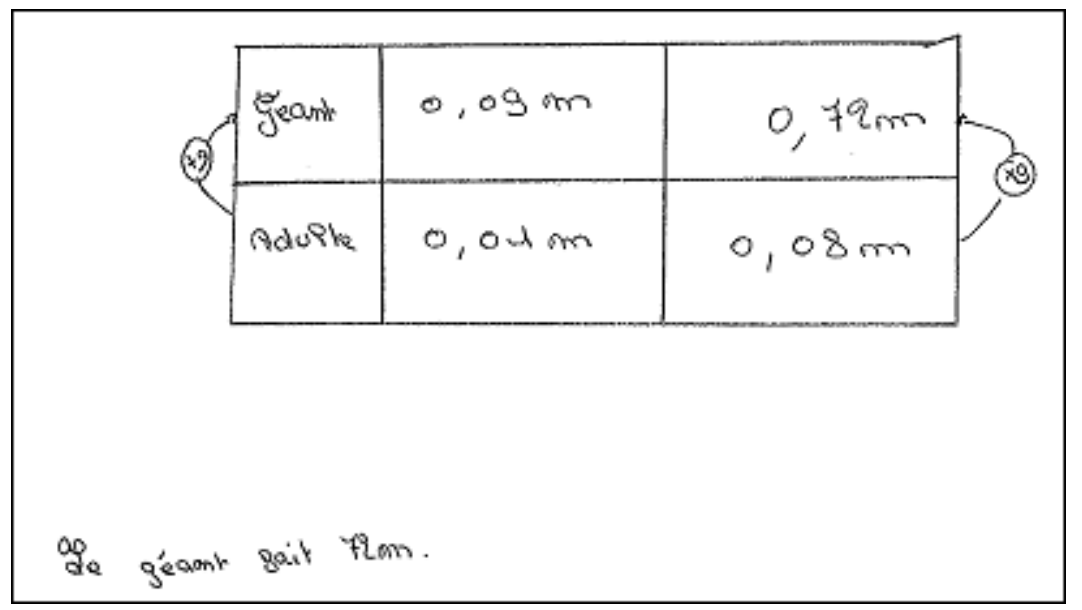

Figure 2. Cahier de l'élève utilisant la technique $\tau_{2 b i s}$

Mais si les techniques mises en œuvre sont toutes du même type, elles ont un autre point commun: elles reposent toutes sur des mesures dans la réalité vécue de l'élève.

\section{Des techniques empiristes}

La faiblesse du milieu dans lequel les élèves sont plongés les conduit à une production endogène des savoirs: c'est dans l'univers de la classe, avec les «moyens de bord », et ce que les élèves savent déjà ou plutôt croient savoir qu'il s'agit de trouver la matière sur laquelle construire une réponse. Or un évènement imprévu s'est produit: ce jour-là il pleut et le professeur porte des bottes. La botte du géant devient alors la botte du professeur et la technique de résolution par report de la hauteur de la botte émerge aussitôt:

P: De quoi aurez-vous besoin pour résoudre ce problème? Qu'allez-vous utilisez? D'après vous ...

E: La taille d'une personne normale.

$\mathrm{P}$ : Tu vas partir de la taille d'une personne normale.

C'est quoi une personne normale?

E: Un adulte.

P: La taille d'un adulte.

E: Et aussi la taille de ta chaussure.

$\mathrm{P}$ : De quoi?

E: La taille de ta chaussure.

$\mathrm{P}$ : À qui?

E: Aux ... oui aux adultes comme ça on peut faire un peu de la proportionnalité.
P: La taille de la chaussure de l'adulte ou du géant?

E: La taille de la chaussure de l'adulte pour faire de la proportionnalité avec ...

Plus tard, ce même élève se fait plus explicite encore lorsqu'il s'agit d'exposer sa technique de résolution lors du premier bilan:

E: D'abord on a mesuré la botte de la maîtresse.

P: Hauteur en fait, c'est ça?

E: La hauteur de la botte de la maîtresse.

P: Hauteur botte d'un adulte donc, oui...

E: Alors ça nous a donné $20 \mathrm{~cm}$; ensuite on a pris la taille moyenne d'un adulte et c'est $1 \mathrm{~m} 70$.

$\mathrm{P}$ : En moyenne un adulte mesure $1 \mathrm{~m} 70$.

E (un autre élève) : $1 \mathrm{~m} 80$ !

$\mathrm{P}$ : On réagira après, on les écoute d'abord.

E: Ensuite on a divisé $1 \mathrm{~m} 70$ par $20 \mathrm{~cm}$ donc on a divisé la taille d'un homme par la hauteur de la botte, ça nous a donné $8,5 \mathrm{~cm}$.

P: Vous cherchiez quoi en faisant ce calcul?

E: Alors sur la photo on voit que la botte fait à peu près la taille des 2 hommes, alors ensuite en sachant qu'une botte mesure $20 \mathrm{~cm}$, on a multiplié 8,5 par $1 \mathrm{~m}$ 70 et ça nous donne la taille du géant.

P : D'accord alors on ne va pas aller plus loin; ça c'était votre stratégie.

Nous voyons ci-dessus comment le principe du recours à l'empirie est légitimé par le professeur qui n'hésitera pas, alors que les élèves seront une dernière fois en recherche individuelle, à les y encourager: « vous pouvez utiliser euh...vos jambes 
ou la hauteur de votre corps pour voir... vous pouvez le faire, vous pouvez mesurer ». Ce sera alors tout l'enjeu de la séance: comment s'y prendre pour déterminer le nombre de reports d'une botte dans un corps? Et, sans surprise c'est cette seule technique du report de la hauteur d'une botte qui sera institutionnalisée lors de la correction collective (voir figure 3), nous y reviendrons.

Nous interprétons ce recours à l'empirie comme une conduite d'évitement de la variabilité. Il est impossible, faute d'avoir interrogé l'élève, de commenter ce que signifie pour lui le mot moyenne qui agit ici comme le marquage d'une spécification de quelque chose de variable. En revanche, la mention de cette notion par le professeur sans être explicitée comme si elle faisait partie d'une culture commune pose question puisqu'elle n'est pas au programme d'enseignement de cette classe. En employant ce mot le professeur semble s'acquitter ainsi du tribut à payer pour prendre en charge, de façon minimaliste, la question de la variabilité de la mesure d'une grandeur. Nous avons montré ailleurs (Chevallard \& Wozniak, 2005; Wozniak, 2005, 2007) les contraintes de niveaux supérieurs qui expliquent la négation de la variabilité dont nous voyons les effets ici: alors que l'élève dit bien qu'il utilise « la hauteur de la botte de la maîtresse ", c'est le professeur luimême qui le reprend et reformule pour poser dans la classe une des variables du système « hauteur botte d'un adulte donc, oui...». Dans cette transformation qui établit une équivalence entre un élément singulier et un élément générique d'une classe, nous retrouvons ce que Brousseau (2005) a repéré comme un possible obstacle épistémologique.

Au-delà, se pose le problème du statut des variables du système. Si la technique décrite par l'élève dans la citation précédente est correcte, les conditions d'autarcie dans lesquelles il est plongé le conduisent à faire un raisonnement qui niant la variabilité fonctionne par rapprochements : il est nécessaire de connaître la hauteur d'une botte, le professeur porte des bottes, il est donc nécessaire de connaître la hauteur des bottes du professeur.

Le statut de l'information - la mesure d'une botte vs la mesure de la botte du professeur - n'étant pas interrogé, ce qui au départ est construit comme une hypothèse (« ne connaissant pas la hauteur d'une botte, nous prendrons comme valeur la mesure de la botte du professeur ») devient un fait: «en sachant qu'une botte mesure $20 \mathrm{~cm} »$. Tout se passe comme si, à un autre niveau d'enseignement, le professeur avait validé le remplacement d'un «si $x=\ldots$ » par un « soit $x=\ldots »$. Or les praxéologies de modélisation portent en elles la nécessité d'expliciter le statut des variables du système: sont-elles des faits, des données de la situation ou des hypothèses au fondement du processus de modélisation?

\section{Les variables du système: hypothèses vs faits}

Nous l'avons dit, une des spécificités du problème proposé à l'étude est l'absence de données numériques dans l'énoncé du problème. Parmi les données observationnelles fournies par la photo nous pouvons citer, par exemple, le fait que deux hommes (adultes) se tiennent debout à côté d'un édifice dont il est dit dans l'énoncé qu'il représente un géant ou encore qu'une partie seulement de la jambe de ce géant est visible sur la photo. Cependant, ces données ne suffisent pas à répondre à la question, il est nécessaire de faire des hypothèses.

Le flou concernant le statut des informations utilisées - données observationnelles ou extrapolées, hypothèses - se concrétise dans l'usage du mot indice. Ainsi, passant dans les rangs pendant le temps de recherche en binôme, le professeur intervient: «Vous êtes bloqués? Faîtes étape par étape, vraiment. Ce que je dois chercher, ce que je sais déjà grâce à l'énoncé, à la photo... Trouvez, cherchez tous les indices que nous donne la photo »

Plus tard, le professeur recourt à une hypothèse en la posant comme un fait. La conservation des proportions entre le géant et un homme adulte qui n'a jamais été interrogée, est pourtant utilisée comme argument pour invalider une réponse d'élève: «Est-ce que c'est possible qu'il fasse $3 \mathrm{~m} 60$ seulement? ça voudrait dire que sa botte est la plus grande partie de son corps. Le géant a les dimensions d'un humain, il n'a pas le pied énormément plus grand que le reste de son corps, c'est les mêmes proportions qu'un adulte, qu'un humain [...] c'est pareil ... que tu sois petite ou grande, le pied a toujours la même proportion par rapport au reste de ton corps ». Or nous savons que c'est faux, les proportions d'un 
enfant de trois ans ne sont pas celles d'un enfant de dix ans ou d'un adulte de trente ans.

L'ambiguité sur le statut des variables du système perdure jusqu'au cours de la correction collective de la résolution du problème. Alors qu'il explicite la distinction entre «ce que l'on sait à partir de la photo » et ce que l'élève "sait par lui-même », le professeur brouille lui-même les cartes en mettant sur un même niveau un fait observé et une mesure hypothétique ${ }^{10}$ :

P: Alors c'est évident mais on va le noter. Je cherche la taille du géant [l'écrit au tableau] vous recopierez la correction après, là on met en commun ensemble. Deuxième étape.

Flore: Je sais qu'un adulte fait environ $1 \mathrm{~m} 70$ et que la botte du géant fait environ $2 \mathrm{~m}$.

$\mathrm{P}$ : Alors d'abord ... Bon alors ce que tu sais grâce à la photo en fait, d'abord. La photo ne te dit pas qu'un adulte fait $1 \mathrm{~m} 80$ c'est toi qui le sais. Ce que la photo, l'énoncé t'apportent comme indice, qu'est-ce que c'est alors, Michel...

Michel: Que le géant porte une botte.

$\mathrm{P}$ : Oui... mais quelque chose qui pourra t'aider dans tes calculs, Chloé...?

Chloé: Que l'homme fait à peu près la taille de la botte.

P : L'homme a environ la hauteur de la botte, ou la même taille que la ... oui et donc là on peut ajouter entre parenthèses ce que tu as dit Flore..., c'est-à-dire la taille d'un adulte.

Flore: $1 \mathrm{~m} 70$.

Ce faisant, le professeur note au tableau: «Je sais que l'homme a environ la même taille que la botte ( 1 m 70).

Or la construction d'un modèle passe par la mise en œuvre d'une dialectique des médias et des milieux qui permette une explicitation de la problématisation au sens de Orange. Si les hypothèses qui fondent le modèle sont énoncées sans être légitimées, sans que leur domaine de validité ne soit exploré alors le processus de modélisation ne s'est pas pleinement développé et l'étape de construction du modèle partiellement réalisée. En conclusion, nous pouvons donc dire que le professeur et ses élèves passent de la définition du système au travail dans un modèle préconstruit. La seule question qui vive dans la classe observée est celle du choix des valeurs des grandeurs utilisées dans l'application du modèle. C'est ainsi que la faiblesse du milieu permettant de déterminer les variables du système sur lesquelles se fonde la construction du modèle va conduire à une évaluation des réponses sur la seule base de l'opinion infondée.

\section{Évaluer les réponses}

Avant la correction collective, le professeur note sous leur dictée les différents résultats des élèves que nous reproduisons ici en les classant par ordre croissant : $8,50 \mathrm{~m} ; 9 \mathrm{~m} ; 10,7 \mathrm{~m} ; 12 \mathrm{~m} ; 13 \mathrm{~m} ; 14 \mathrm{~m}$; $14,45 \mathrm{~m} ; 16,20 \mathrm{~m} ; 18 \mathrm{~m} ; 36 \mathrm{~m} ; 90 \mathrm{~m}$. Une mise au point est faite en prélude à la discussion sur les conditions de l'évaluation des résultats sans révéler les critères qui seront utilisés:

«Bien, avant de le corriger il faut savoir que personne d'entre nous, ni vous, ni moi ne pouvons connaître la vraie taille du géant. Je ne suis pas allée le mesurer en vrai. Je ne sais pas. Donc on ne peut trouver qu'une réponse approximative, d'accord? À peu près... On pourra accepter ou refuser certaines réponses, on verra lesquelles et pourquoi. Mais je ne suis pas en mesure de vous donner la taille exacte du géant. En revanche il y a des tailles que l'on peut éliminer de façon sûre et d'autres que l'on peut garder, on verra lesquelles. »

Les discussions sur la validité des résultats tournent autour des techniques utilisées pour déterminer le nombre de reports de la hauteur d'une botte sur le corps d'un homme adulte ou d'un élève. Finalement, le professeur tranche en imposant le recours à l'empirie :

P : Qui vient m'aider là, Flore, viens ...

$\mathrm{P}$ : Alors, on va voir avec mes bottes combien de fois on va mettre la hauteur de mes bottes sur moi. Tiens, alors tu mesures la hauteur de ma botte. Alors 23, on va reporter ça à chaque fois, 2 bottes, ...

E: 3

$\mathrm{P}: 4$

E: 5

$\mathrm{P}:$ Monte la, pose la sur mon doigt.

E: 6

P: Et la tête, ça fait 7.

$\mathrm{E}$ : Donc 7 fois 2, 14 .

$\mathrm{P}$ : Donc à peu près 7 fois. Mais c'est pas exact, c'est à peu près. On ne sait pas si les bottes du géant, ils ont... c'est la même proportion que les miennes sur moi, mais 
c'est à peu près 7 . Mais on peut les mettre 10 fois, c'est juste aussi vu qu'on ne sait pas exactement quelle est la taille de ses bottes.

C'est donc à l'aune de cette valeur $14 \mathrm{~m}$ et des mesures réalisées sur le professeur que vont être évaluées les réponses des élèves:

P: 9, c'est pas assez, hein. C'est trop petit. 12 à mon avis c'est limite, limite; 10 ce n'est pas bon. 18,50 non plus, 12 hum... Bon, on peut accepter. Donc, en fait on cherche un ordre de grandeur donc ça se trouve entre 13 et 25 centimètres ... euh ... 13 et 25 mètres. Un ordre de grandeur, il n'y a pas de réponse exacte. Alors est-ce que dans ce problème, il y avait une seule façon de le résoudre?

E: Non.

$\mathrm{P}$ : Est-ce qu'il y a une réponse exacte?

E: Non.

P: Non. D'accord?

En réalité, la classe reste dans le monde de l'arbitraire car aucun élément objectif ne permet, ici, de proposer un tel intervalle de valeurs. Remarquons simplement qu'avec un géant ayant une taille de $25 \mathrm{~m}$ et des bottes de $2 \mathrm{~m}$, cela représenterait 11,5 reports d'une botte dans un corps. En gardant ce rapport, sur un homme de 1,80 m cela donnerait des bottes de 15,6 cm. Par ailleurs, ce que le professeur identifie comme différentes façons de résoudre le problème ne sont en réalité que des spécifications du même type de technique $\tau_{1}$. Leur différence spécifique se situant dans le mode de détermination du nombre de reports ou la valeur des mesures utilisées pour la hauteur d'une botte ou la taille d'un homme adulte. C'est ce qui sera institutionnalisé au travers de la correction collective du problème.

\section{Une institutionnalisation qui laisse dans l'ombre la construction du modèle}

La correction construite collectivement (figure 3) présente le travail dans le modèle sans révéler sur quoi se construit le modèle utilisé.

Si le modèle de la botte utilisée comme étalon est explicité, la correction laisse implicite les hypothèses faites pour construire une réponse. En particulier l'hypothèse fondatrice du modèle utilisé: le corps du géant est un agrandissement (homothétique) du corps d'un être humain (H2). Notons que les proportions d'un enfant ne sont pas identiques à celles d'un adulte et la correction entretient une certaine ambiguité sur ce point en évoquant " un corps humain », ce qui semble signifier qu'il est fait l'hypothèse que les proportions d'un être humain à un autre sont conservées (H1).

La variété des réponses apparait comme le fruit de la variété des reports $(7,8,9,10)$ sur un corps de la hauteur d'une botte sans que soit explicité que cette variété dépend de la mesure de la hauteur d'une botte prise pour étalon et de la taille du corps sur lequel ce report est effectué. À titre d'exemple, sur un homme de $1,80 \mathrm{~m}$ les reports $7,8,9$ et 10 correspondraient à des bottes de hauteur respective $25,7 \mathrm{~cm} ; 22,5 \mathrm{~cm}$; $20 \mathrm{~cm}$ et $18 \mathrm{~cm}$. Par ailleurs, la variété des réponses liée au choix de l'estimation de la hauteur de la botte du géant lui-même $(2 \mathrm{~m})$ en référence à la variété possible des tailles des hommes sur la photo n'est pas explicitée. Enfin, notons qu'il est donné le même statut de validité à un report effectué sur un corps d'enfant et sur un schéma sans que ne soit mentionné le domaine de validité d'une telle procédure: il est indispensable que le schéma soit bien une représentation homothétique du corps d'un homme adulte!

\section{Quel enjeu didactique?}

L'enjeu du savoir se révèle au travers du discours technologique qui met à jour, décrit, explicite, justifie, questionne et valide les connaissances utilisées. C'est l'institutionnalisation qui permet à la classe de distinguer le contingent du nécessaire dans l'élaboration de l'organisation mathématique visée. Il faut des mots, des notations, des ostensifs (Bosch \& Chevallard, 1999) pour que la classe se dise à ellemême les savoirs qu'elle a collectivement construits et qui intégreront une culture partagée. Il apparait que, pour le professeur observé ici, l'enjeu de savoir dans l'étude de ce problème n'est pas la démarche de modélisation mais l'obtention de la réponse par application de la proportionnalité. Le seul travail à réaliser avec les élèves portait sur le choix des valeurs numériques à utiliser dans la mise en œuvre d'un modèle préconstruit. L'enjeu réel est bien la consolidation de connaissances déjà institutionnalisées et ne peut être l'acquisition de praxéologies de modélisation, objet de savoir non identifié par ce professeur. 


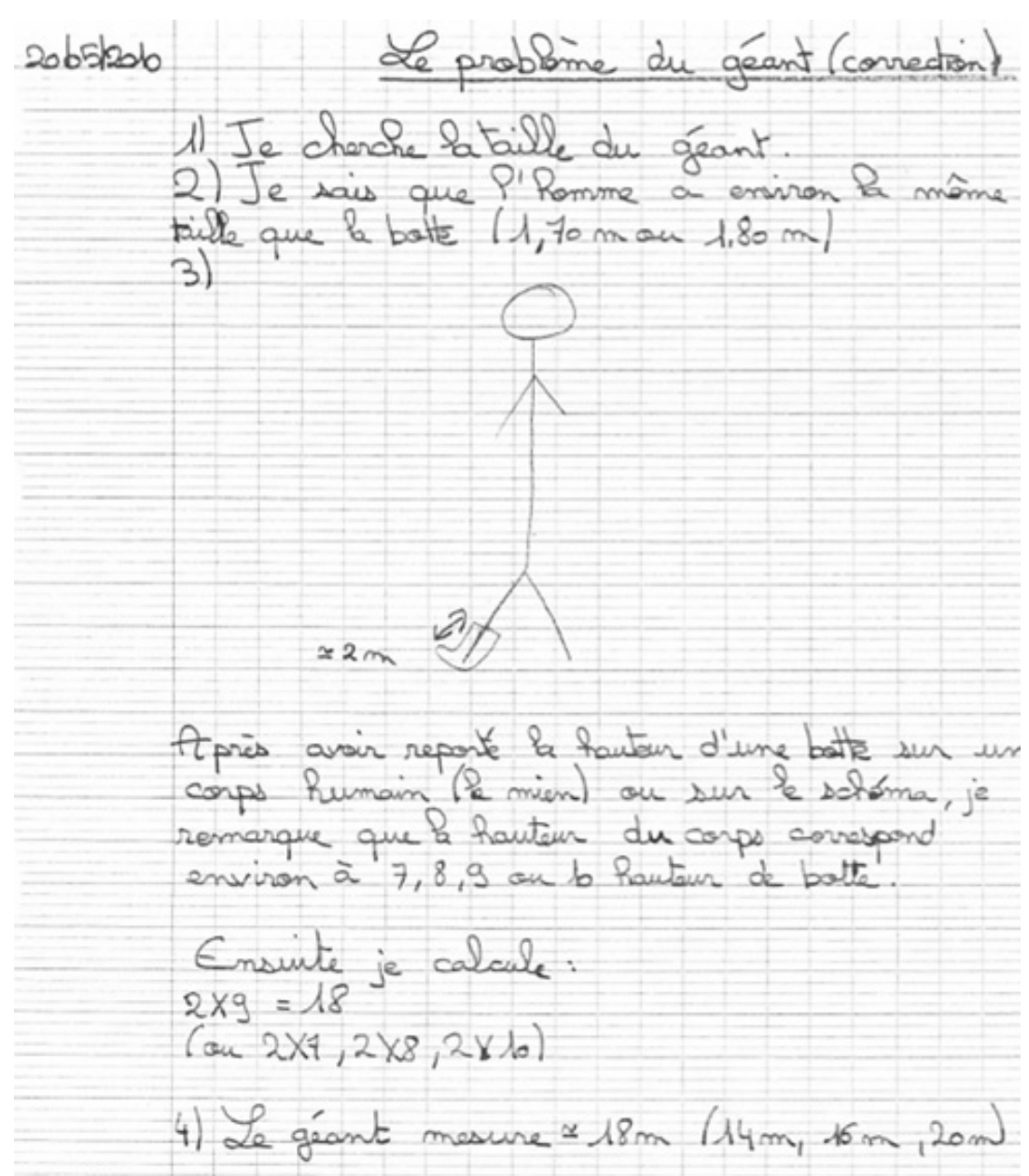

Figure 3. Correction construite collectivement

Nous avons vu le professeur enseigner la réponse et non comment penser le problème. Les élèves ne rencontrent pas leur ignorance par rapport à la construction d'un modèle mais seulement par rapport à la production des données.

Comme le souligne Brousseau (1998), pour que les savoirs deviennent des moyens économiques permettant de résoudre de nouveaux problèmes, il est nécessaire de sortir du contingent. Si le recours à l'empirie peut être légitime pour produire une réponse à une question, il ne peut se suffire à lui-même. Rencontrer le mathématique c'est révéler le générique derrière la spécificité de la situation étudiée:

« Le professeur doit donc simuler dans sa classe une micro-société scientifique s'il veut que les connaissances soient des moyens économiques pour poser de bonnes questions et pour trancher des débats, s'il veut que les langages soient des moyens de maîtriser des situations de formulation et que les démonstrations soient des preuves. Mais il doit aussi donner les moyens à ses élèves de retrouver dans cette histoire particulière qu'il leur a fait vivre, ce qu'est le savoir culturel et communicable qu'on a voulu leur enseigner. » (Brousseau, 1998, p. 49-50).

Dans la classe observée une certaine praxis relevant de la modélisation s'est donnée à voir mais très peu de logos associé: un modèle est utilisé et fonctionne sans discuter sa légitimité ou son domaine de validité. Au regard du modèle épistémologique de référence que nous utilisons, nous dirons que les praxéologies liées à la construction d'un modèle mathématique sont muettes. Il semble, en effet, que 
se construisent essentiellement des rapports d'action, parfois de façon partielle, des rapports de formulation. Quant aux rapports de validation, propres aux praxéologies fortes, nous n'en voyons aucune trace.

Nous l'avons dit deux corps de savoirs étaient en jeu pour résoudre ce problème: les praxéologies liées au processus de modélisation et celles liées à l'étude de situations pouvant être représentées par le modèle de la proportionnalité. La rapidité avec laquelle un élève fait mention de la proportionnalité et l'absence de question sur ce point de la part des autres élèves, laissent penser que, dans cette classe, la proportionnalité est un savoir institutionnalisé et mobilisable à volonté pour traiter une nouvelle situation. En revanche, concernant le processus de modélisation, la direction de l'étude du professeur ne pouvait permettre la dévolution d'une première rencontre car la reconnaissance, par le professeur, d'un besoin de savoir est une condition minimale:

« On peut confier la première rencontre avec le savoir nouveau à une situation adidactique qui "porte" le savoir nouveau sous la forme de connaissances à mettre en œuvre pour traiter le problème, la production des connaissances par les élèves pourra se faire à condition de faire dévolution de cette situation aux élèves » (Perrin-Glorian \& Hersant, 2003, p. 241-242).

Or, grâce aux réponses de ce professeur à nos questionnaires, nous savons qu'il n'avait pas identifié ce problème comme un problème de modélisation, mais comme un "problème inédit dans sa forme » puisque «tous les indices, sur lesquels les élèves devront s'appuyer pour résoudre le problème, se trouvent dans la photo et non pas dans l'énoncé ». Là où le professeur observé ne voit qu'une question de forme, il y a bien une question d'ordre épistémologique. Faute d'avoir identifié le processus de modélisation comme un savoir à enseigner, ce qui a été l'enjeu réel d'enseignement est l'initiation du mode de production de la réponse du problème, c'est bien une praxis qui a été initiée. Nous pourrions donc dire, concernant les praxéologies de modélisation, que le professeur observé a organisé un moment de première rencontre sans dévolution. Ce faisant il a instauré dans la classe ce que Perrin-Glorian et Hersant (2003) appellent un méso-contrat d'initiation dans lequel « on a une transmission directe du savoir, par ostension. Le savoir montré par le profes- seur doit devenir une connaissance de l'élève. » (Perrin-Glorian \& Hersant (2003), p. 242).

Les praxéologies de modélisation vivant dans la classe ne pouvaient être alors que des praxéologies muettes.

\section{Conclusion}

L'étude de cas dont nous venons de rendre compte a permis de mettre à jour les praxéologies spontanées de modélisation du professeur observé: le recours à l'empirie au service d'une production endogène des savoirs; une organisation mathématique fondée sur le travail dans le modèle plutôt que sur la construction du modèle révélée par la confusion entre hypothèses et faits; un processus de modélisation qui n'est pas enjeu didactique conduisant à la mise en ouvre de praxéologies muettes; une conduite de l'étude qui enseigne la réponse plutôt que les moyens de la produire.

Nous faisons l'hypothèse que ce que nous avons observé n'est pas le seul fruit de la singularité d'un professeur particulier mais relève davantage d'un état de la profession. Pour illustrer ce point de vue nous allons aborder un aspect que nous n'avons pas évoqué jusque-là : les professeurs des écoles sont pluridisciplinaires, en particulier ils enseignent les sciences où le processus de modélisation n'est pas étranger à la démarche d'investigation entrée en vigueur dans les programmes d'enseignement en France en 2002 (BOEN HS n ${ }^{\circ} 1$ du 14 février 2002). C'est ainsi qu'au moment de notre observation, la rubrique relative aux « sciences physiques et la technologie » dans les programmes d'enseignement de la classe observée stipulait:

«Observation, questionnement, expérimentation et argumentation pratiqués, par exemple, selon l'esprit de la Main à la pâte sont essentiels pour atteindre ces buts; c'est pourquoi les connaissances et les compétences sont acquises dans le cadre d'une démarche d'investigation qui développe la curiosité, la créativité, l'esprit critique et l'intérêt pour le progrès scientifique et technique. » (Ministère de l'Éducation Nationale, 2008, p. 24)

Nous ne savons rien des pratiques effectives d'enseignement des sciences du professeur observé, cependant nous savons que ce professeur des écoles, 
dont l'ancienneté est de moins de cinq ans, a été formé dans un IUFM. Nous pouvons donc supposer qu'il a connaissance de la démarche d'investigation en sciences et qu'il sait la nécessité d'expliciter et questionner des hypothèses, par exemple. Un constat semble alors s'imposer: il n'y a pas de transfert «spontané » de praxéologies d'une discipline scolaire à une autre, fussent-elles si proches.

Ainsi, au-delà des besoins de connaissances mathématiques qui se révèlent par des praxéologies de modélisation muettes, apparaissent des besoins de praxéologies didactiques. Praxéologies qui extirpent les savoirs du particularisme des problèmes du réel où ils sont rencontrés et qui, pour reprendre les mots de Brousseau (1998), révèlent les savoirs comme des moyens économiques permettant de résoudre de nouveaux problèmes. En effet,

« Ce qui est montré, enseigné et retenu est moins essentiel que la manière dont on leur a fait considérer les problèmes, dont on les a conduits à chercher à les résoudre... [...] C'est l'universalité de ces manières de voir, d'agir, de chercher... que décrète l'institutionnalisation, l'expression d'un usage commun auquel désormais doivent se contraindre les élèves. » (Sarrazy, 2007, p. 39-40).

Or si la notion de problématisation de Orange $(2005,2007)$ nous intéresse c'est que nous y trouvons, dans le champ de la didactique des sciences de la Vie et de la Terre, des questions qui se posent dans celui de la didactique des mathématiques. En particulier, le rôle du discours technologique dans la part de construction du modèle apparait fondamental dans l'évaluation des praxéologies de modélisation.

Ainsi, si l'identification de certaines praxéologies didactiques spécifiques à la démarche d'investigation est une des conditions pour que vivent dans les classes des praxéologies de modélisation mathématiques, alors un dialogue entre didacticiens des disciplines scientifiques scolaires devrait avantageusement y contribuer. 


\section{NOTES}

1. Assude, Mercier, et Sensevy (2007) proposent l'adjectif muet comme alternative à invisible précisant que leur vocabulaire n'est pas encore stabilisé.

2. Copyright Richard Phillips (2001-2009): www.problempictures.co.uk

3. Une homothétie se définit par son centre $\mathrm{O}$ et son rapport $\mathrm{k}$. Elle transforme un point $\mathrm{P}$ en un point P'sur la droite (OP) tel que $\overrightarrow{O P^{\prime}}=k \times \overrightarrow{O P}$. Si une figure est un agrandissement homothétique d'une autre, elle a donc la même forme et toutes ses dimensions sont multipliées par la même valeur (le coefficient d'agrandissement) par rapport à la figure initiale.

4. La longueur du pied est confondue avec la semelle de la chaussure, néanmoins nous distinguons les deux cas puisque les discours technologiques associés aux techniques qui les utilisent font la distinction.

5. D'après le site de l'INSEE, la taille moyenne d'un homme en France entre 18 et 65 ans est de 1,75 m : http://www.insee.fr/fr/themes/document.asp?ref_id=ip1123\&reg_id=0

6. Notons que le choix des exemples de Orange (2005) et de Chevallard (1999), par exemple, expriment la volonté de proposer un cadre théorique qui dépasse le champ de recherche d'où il est issu, la didactique des sciences de la Vie et de la Terre pour l'un, la didactique des mathématiques pour l'autre.

7. Nous parlons de saturation du registre empirique lorsque le maître ou les élèves introduisent de nouvelles observations, expériences ou documents relatifs à des observations ou expériences. Lorsque ce sont des documents sur des modèles (documentaires par exemple) qui sont introduits, c'est le registre des modèles qui est saturé.

8. Distinction que Sarrazy (2007) n'hésite pas à qualifier d' « un des apports majeurs » à l'enseignement des mathématiques de la théorie des situations didactiques due à Guy Brousseau.

9. Les membres du groupe ACODIS ayant participé à l'étude en 2009-2010 sont : R. Adjiage, T. Beliaeva, N. Gavens, A. Jarlegan, J.-C. Rauscher, M.-J. Remigy, M. Weisser, F. Wozniak.

10. Les prénoms ont été changés dans cette citation.

\section{RÉFÉRENCES}

Assude, T., Mercier, A., \& Sensevy, G. (2007). L'action didactique du professeur dans la dynamique des milieux. Recherches en Didactique des Mathématiques, 27(2), p. 221-252.

Bourny, G., Dupé, C., Robin, I., \& Rocher, T. (2001). Les élèves de 15 ans, premiers résultats d'une évaluation internationale des acquis des élèves (PISA).Note d'information 01.52. Ministère de l'Éducation nationale. ftp:// trf.education.gouv.fr/pub/edutel/dpd/ni0152.pdf

Bosch, M. \& Chevallard, Y. (1999). La sensibilité de l'activité mathématique aux ostensifs. Objet d'étude et problématique. Recherches en Didactique des Mathématiques, 19(1), p. 77-124.

Brousseau, G. (1998). Théorie des situations didactiques. Grenoble: La pensée sauvage. 
Brousseau, G. (2005). Situations fondamentales et processus génétique de la statistique. In A. Mercier \& C. Margolinas (Éds.), Balises pour la didactique des mathématiques (p. 165-194). Grenoble: La pensée sauvage.

Chevallard, Y. (1989). Le passage de l'arithmétique à l'algèbre dans l'enseignement des mathématiques au collège. Deuxième partie. Perspectives curriculaires : la notion de modélisation. Petit x, 19, p. 43-72.

Chevallard, Y. (1999). L'analyse des pratiques enseignantes en théorie anthropologique du didactique. Recherches en Didactique des Mathématiques, 19(2), p. 221-266.

Chevallard, Y. (2002). Organiser l'étude. Écologie et régulation. In J.-L. Dorier, M. Artaud, M. Artigue, R. Berthelot, \& R. Floris (Éds.), Actes de la $11^{e}$ école d'été de Didactique des Mathématiques (p. 41-56). Grenoble: La Pensée Sauvage.

Chevallard, Y. (2010). La didactique dites-vous? Éducation E didactique, 4(1), p. 139-148.

Chevallard, Y. (2011). La notion d'ingénierie didactique, un concept à réfonder. Questionnement et éléments de réponse à partir de la TAD. In C. Margolinas, M. Abboud-Blanchard, L. Bueno-Ravel, N. Douek, A. Fluckiger, P. Gibel, F. Vandebrouck, F. Wozniak (coord.), En amont et en aval des ingénieries didactiques. XVéécole d'été de didactique des mathématiques. (p. 81-108). Grenoble: La pensée sauvage.

Chevallard, Y., \& Wozniak, F. (2005) Enseigner la statistique au secondaire. Entre genre prochain et différence spécifique. In A. Mercier, \& C. Margolinas (Éds.), Balises pour la didactique des mathématiques (p. 195-218). Grenoble: La pensée sauvage.

Ladage, C., \& Chevallard, Y. (2011). Enquêter avec l'internet. Études pour une didactique de l'enquête. Éducation \& didactique, 5(2), p. 85-115.

Ministère de l'Éducation nationale (2008). Horaires et programmes d'enseignement de l'école primaire. BOEN $\mathrm{n}^{\circ}$ 3, 19 juin 2008, nº́cial.

Orange, C. (2005). Problématisation et conceptualisation en sciences et dans les apprentissages scientifiques. Les Sciences de l'éducation, Pour l'ère nouvelle, 38(3), p. 69-93. http://creas.educ.usherbrooke.ca/Archives/ OrangeChr_Lecture.pdf

Orange, C. (2007). Quel Milieu pour l'apprentissage par problématisation en sciences de la vie et de la terre? Éducation E didactique, 1(2), p. 37-56

Perrin-Glorian, M.-J., \& Hersant, M. (2003). Milieu et contrat didactique, outils pour l'analyse de séquences ordinaires. Recherches en Didactique des Mathématiques, 23(2), p. 217-276.

Rocard, M., Csermely, P., Jorde, D., Lenzen, D, Walberf-Henrikson, H., \& Hemmo,V. (2007). L'enseignement scientifique aujourd'hui: Une pédagogie renouvelée pour l'avenir de l'Europe. Commissions Européennes: Belgique. http://ec.europa.eu/research/science-society/document_library/pdf_06/ report-rocard-on-science-education_fr.pdf

Sarrazy, B. (2007). Ostension et dévolution dans l'enseignement des mathématiques. Éducation E didactique, 1(3), p. 31-46. 
Floriane Wozniak

Wozniak, F. (2005). Conditions et contraintes de l'enseignement de la statistique en classe de seconde générale. Un repérage didactique. Thèse de doctorat, Université Lyon 1, Lyon. http://tel.archives-ouvertes.fr/ docs/00/06/41/60/PDF/these wozniak floriane.pdf

Wozniak, F. (2007). Conditions and constraints in the teaching of statistics: the scale of levels of determination. In D. Pitta-Pantazi, \& G. Philippou (Éds.), Proceedings of the European Society for Research in Mathematics Education. CERME 5 (p. 1808-1818). Larnaca: University of Cyprius. http://ermeweb.free.fr/CERME\%205/ CERME5\%20Proceedings\%20Book.pdf 